\title{
ROUGH SINGULAR INTEGRALS ON PRODUCT SPACES
}

\author{
AHMAD AL-SALMAN and HUSSAIN AL-QASSEM
}

Received 26 December 2003

We study the mapping properties of singular integral operators defined by mappings of finite type. We prove that such singular integral operators are bounded on the Lebesgue spaces under the condition that the singular kernels are allowed to be in certain block spaces.

2000 Mathematics Subject Classification: 42B20, 42B15, 42B25.

1. Introduction and results. For $n \in \mathbb{N}, n \geq 2$, let $K(\cdot)$ be a Calderón-Zygmund kernel defined on $\mathbb{R}^{n}$, that is,

$$
K_{\Omega}(y)=\Omega(y)|y|^{-n},
$$

where $\Omega \in L^{1}\left(\mathbf{S}^{n-1}\right)$ is a homogeneous function of degree zero that satisfies

$$
\int_{\mathbf{S}^{n-1}} \Omega(y) d \sigma(y)=0
$$

with $d \sigma(\cdot)$ being the normalized Lebesgue measure on the unit sphere.

Let $B_{n}(0,1)$ be the unit ball centered at the origin in $\mathbb{R}^{n}$. For a $\mathscr{C}^{\infty}$ mapping $\Phi$ : $B_{n}(0,1) \rightarrow \mathbb{R}^{d}, d \geq 1$, consider the singular integral operator

$$
T_{\Phi, \Omega} f(x)=\text { p.v. } \int_{B_{n}(0,1)} f(x-\Phi(y)) K_{\Omega}(y) d y,
$$

where, p.v. denotes the principal value.

It is known that if $\Phi$ is of finite type at 0 (see Definition 2.2) and $\Omega \in \mathscr{C}^{1}\left(\mathbf{S}^{n-1}\right)$, then $T_{\Phi, \Omega}$ is bounded on $L^{p}$ for $1<p<\infty$ [15]. Moreover, it is known that $T_{\Phi, \Omega}$ may fail to be bounded on $L^{p}$ for any $p$ if the finite-type condition is removed. In [8], Fan et al. showed that the $L^{p}$ boundedness of the operator $T_{\Phi, \Omega}$ still holds if the condition $\Omega \in \mathscr{C}^{1}\left(\mathbf{S}^{n-1}\right)$ is replaced by the weaker condition $\Omega \in L^{q}\left(\mathbf{S}^{n-1}\right)$ for some $q>1$. Subsequently, the $L^{p}$ $(1<p<\infty)$ boundedness of $T_{\Phi, \Omega}$ was established under conditions much weaker than $\Omega \in L^{q}\left(\mathbf{S}^{n-1}\right)[1,6]$. In particular, Al-Qassem et al. [1] established the $L^{p}$ boundedness of $T_{\Phi, \Omega}$ under the condition that the function $\Omega$ belongs to the block space $B_{q}^{0,0}\left(\mathbf{S}^{n-1}\right)$ introduced by Jiang and Lu in (see [14]). In fact, they proved the following theorem.

THEOREM 1.1. Let $T_{\Phi, \Omega}$ be given by (1.3). Suppose that $\Omega \in B_{q}^{0,0}\left(\mathbf{S}^{n-1}\right)$ for some $q>1$. If $\Phi$ is of finite type at 0 , then for $1<p<\infty$ there exists a constant $C_{p}>0$ such that

$$
\left\|T_{\Phi, \Omega} f\right\|_{L^{p}\left(R^{d}\right)} \leq C_{p}\|f\|_{L^{p}\left(R^{d}\right)}
$$

for any $f \in L^{p}\left(\mathbb{R}^{d}\right)$. 
It should be pointed out here that the condition $\Omega \in B_{q}^{0,0}\left(\mathbf{S}^{n-1}\right)$ in Theorem 1.1 was recently proved to be nearly optimal. In fact, Al-Qassem et al. [2] showed that if the condition $\Omega \in B_{q}^{0,0}\left(\mathbf{S}^{n-1}\right)$ is replaced by $\Omega \in B_{q}^{0, v}\left(\mathbf{S}^{n-1}\right)$ for some $v<0$, then the corresponding classical Calderón-Zygmund operator

$$
T_{\Omega} f(x)=\text { p.v. } \int_{\mathbb{R}^{n}} f(x-y) K_{\Omega}(y) d y
$$

may fail to be bounded on $L^{p}$ at any $1<p<\infty$.

Fefferman [11] and Fefferman and Stein [12] studied singular integrals on product domains. Namely, they studied operators of the form

$$
\left(P_{\Omega} f\right)(x, y)=\text { p.v. } \iint_{\mathbb{R}^{n} \times \mathbb{R}^{m}} f(x-u, y-v) K_{\Omega}(u, v) d u d v,
$$

where $n, m \geq 2$,

$$
\begin{gathered}
K_{\Omega}(u, v)=\Omega\left(|u|^{-1} u,|v|^{-1} v\right)|u|^{-n}|v|^{-m}, \\
\Omega \in L^{1}\left(\mathbf{S}^{n-1} \times \mathbf{S}^{m-1}\right), \Omega(t x, s y)=\Omega(x, y) \quad \text { for any } t, s>0, \\
\int_{\mathbf{S}^{n-1}} \Omega(u, \cdot) d \sigma(u)=0, \quad \int_{\mathbf{S}^{m-1}} \Omega(\cdot, v) d \sigma(v)=0 .
\end{gathered}
$$

In [12], it was shown that $P_{\Omega}$ is bounded on $L^{p}\left(\mathbb{R}^{n+m}\right)$ for $1<p<\infty$ if $\Omega$ satisfies some regularity conditions. Subsequently, the $L^{p}(1<p<\infty)$ boundedness of $P_{\Omega}$ was established under weaker conditions on $\Omega$, first in [7] for $\Omega \in L^{q}\left(\mathbf{S}^{n-1} \times \mathbf{S}^{m-1}\right)$ with $q>1$ and then in [9] for $\Omega \in \bigcup_{q>1} B_{q}^{0,1}\left(\mathbf{S}^{n-1} \times \mathbf{S}^{m-1}\right)$ which contains $\bigcup_{q>1} L^{q}\left(\mathbf{S}^{n-1} \times \mathbf{S}^{m-1}\right)$ as a proper subspace, where $B_{q}^{0,1}$ represents a special class of block space on $\mathbf{S}^{n-1} \times \mathbf{S}^{m-1}$; for $p=2$, it was proved by Jiang and Lu in [13]). The definition of block spaces will be recalled in Section 2 (see Definitions 2.2 and 2.3).

The analogue of the operators $T_{\Phi, \Omega}$ in (1.3) on product domains is defined as follows.

For $N, M \in \mathbb{N}$, let $\Phi: B_{n}(0, r) \rightarrow \mathbb{R}^{N}$ and $\Psi: B_{m}(0, r) \rightarrow \mathbb{R}^{M}$ be $\mathscr{C}^{\infty}$ mappings. Define the singular integral operator $P_{\Omega, \Phi, \Psi}$ by

$$
\left(P_{\Omega, \Phi, \Psi} f\right)(x, y)=\text { p.v. } \iint_{B_{n}(0,1) \times B_{m}(0,1)} f(x-\Phi(u), y-\Psi(v)) K_{\Omega}(u, v) d u d v
$$

Using the ideas developed in [4, 8], we can easily show that $P_{\Omega, \Phi, \Psi}$ is bounded on $L^{p}$ $(1<p<\infty)$ provided that $\Phi$ and $\Psi$ are of finite type at 0 and $\Omega \in L^{q}\left(\mathbf{S}^{n-1} \times \mathbf{S}^{m-1}\right)$ for some $q>1$. However, the natural question that arises here is as follows.

QUESTION 1.2. Suppose that $\Omega \in \bigcup_{q>1} B_{q}^{0,1}\left(\mathbf{S}^{n-1} \times \mathbf{S}^{m-1}\right)$ and $\Phi$ and $\Psi$ are of finite type at 0 . Is the operator $P_{\Omega, \Phi, \Psi}$ bounded on $L^{p}(1<p<\infty)$ ?

In this paper, we will answer this question in the affirmative. In fact, we have the following theorem. 
THEOREM 1.3. Let $P_{\Omega, \Phi, \Psi}$ be given by (1.8). Suppose that $\Omega \in B_{q}^{0,1}\left(\mathbf{S}^{n-1} \times \mathbf{S}^{m-1}\right)$ for some $q>1$. If $\Phi$ and $\Psi$ are of finite type at 0 , then for $1<p<\infty$ there exists a constant $C_{p}>0$ such that

$$
\left\|P_{\Omega, \Phi, \Psi}(f)\right\|_{L^{p}\left(\mathbb{R}^{N} \times \mathbb{R}^{M}\right)} \leq C_{p}\|f\|_{L^{p}\left(\mathbb{R}^{N} \times \mathbb{R}^{M}\right)}
$$

for any $f \in L^{p}\left(\mathbb{R}^{N} \times \mathbb{R}^{M}\right)$.

Regarding the condition $\Omega \in B_{q}^{0,1}\left(\mathbf{S}^{n-1} \times \mathbf{S}^{m-1}\right)$ in Theorem 1.3, we should remark here that in a recent paper [5], Al-Salman was able to obtain a similar result to that in [2]. More precisely, Al-Salman showed that the size condition $\Omega \in B_{q}^{0,1}\left(\mathbf{S}^{n-1} \times \mathbf{S}^{m-1}\right)$ is sharp in the sense that if $\Omega \in B_{q}^{0,1}\left(\mathbf{S}^{n-1} \times \mathbf{S}^{m-1}\right)$ is replaced by $\Omega \in B_{q}^{0,1-\varepsilon}\left(\mathbf{S}^{n-1} \times \mathbf{S}^{m-1}\right)$ for some $\varepsilon>0$, then the operator $P_{\Omega}$ may fail to be bounded on $L^{p}$ for any $p$.

Also, in this paper we will give a similar result for the truncated singular integral operator

$$
P_{\Omega, \Phi, \Psi}^{*} f(x, y)=\sup _{\varepsilon_{1}, \varepsilon_{2}>0}\left|\iint_{E\left(\varepsilon_{1}, \varepsilon_{2}, r\right)} f(x-\Phi(u), y-\Psi(v)) K_{\Omega}(u, v) d u d v\right|,
$$

where $E\left(\varepsilon_{1}, \varepsilon_{2}, r\right)=\left\{(u, v) \in \mathbb{R}^{n} \times \mathbb{R}^{m}: \varepsilon_{1} \leq|u|<r, \varepsilon_{2} \leq|v|<r\right\}, x \in \mathbb{R}^{N}$ and $y \in \mathbb{R}^{M}$. In fact, we have the following.

THEOREM 1.4. Let $P_{\Omega, \Phi, \Psi}^{*}$ be given by (1.1) with $r=1$. Suppose that $\Omega \in B_{q}^{0,1}\left(\mathbf{S}^{n-1} \times\right.$ $\mathrm{S}^{m-1}$ ) for some $q>1$. If $\Phi$ and $\Psi$ are of finite type at 0 , then for $1<p<\infty$ there exists $a$ constant $C_{p}>0$ such that

$$
\left\|P_{\Omega, \Phi, \Psi}^{*}(f)\right\|_{L^{p}\left(\mathbb{R}^{N} \times \mathbb{R}^{M}\right)} \leq C_{p}\|f\|_{L^{p}\left(\mathbb{R}^{N} \times \mathbb{R}^{M}\right)}
$$

for any $f \in L^{p}\left(\mathbb{R}^{N} \times \mathbb{R}^{M}\right)$.

It is worth pointing out that, as in the one-parameter setting, we can show that the $L^{p}$ boundedness of the operators $P_{\Omega, \Phi, \Psi}$ and $P_{\Omega, \Phi, \Psi}^{*}$ may fail for any $p$ if at least one of the mappings $\Phi$ and $\Psi$ is not of finite type at 0 .

2. Some definitions and lemmas. We start by the following definition.

DEFINITION 2.1. Let $U$ be an open set in $\mathbb{R}^{n}$ and $\Theta: U \rightarrow \mathbb{R}^{d}$ a smooth mapping. For $x_{0} \in U$, it is said that $\Theta$ is of finite type at $x_{0}$ if, for each unit vector $\eta$ in $\mathbb{R}^{d}$, there is a multi-index $\alpha$ so that

$$
\partial_{x}^{\alpha}[\Theta(x) \cdot \eta]_{x=x_{0}} \neq 0
$$

DEFINITION 2.2. For $1<q \leq \infty$, it is said that a measurable function $b(x, y)$ on $\mathbf{S}^{n-1} \times \mathbf{S}^{m-1}$ is a $q$-block if it satisfies the following:

(i) $\operatorname{supp}(b) \subseteq I$, where $I$ is a cap on $\mathbf{S}^{n-1} \times \mathbf{S}^{m-1}$, that is,

$$
I=\left\{x^{\prime} \in \mathbf{S}^{n-1}:\left|x^{\prime}-x_{0}^{\prime}\right|<\alpha\right\} \times\left\{y^{\prime} \in \mathbf{S}^{m-1}:\left|y^{\prime}-y_{0}^{\prime}\right|<\beta\right\}
$$

for some $\alpha, \beta>0, x_{0}^{\prime} \in \mathbf{S}^{n-1}$, and $y_{0}^{\prime} \in \mathbf{S}^{m-1}$;

(ii) $\|b\|_{L^{q}} \leq|I|^{-1 / q^{\prime}}$, where $1 / q+1 / q^{\prime}=1$. 
Definition 2.3. The class $B_{q}^{0,1}\left(\mathbf{S}^{n-1} \times \mathbf{S}^{m-1}\right), 1<q \leq \infty$, consists of all functions $\Omega \in L^{1}\left(\mathbf{S}^{n-1} \times \mathbf{S}^{m-1}\right)$ of the form $\Omega=\sum_{\mu=1}^{\infty} c_{\mu} b_{\mu}$, where each $c_{\mu}$ is a complex number; each $b_{\mu}$ is a $q$-block supported on a cap $I_{\mu}$ on $\mathbf{S}^{n-1} \times \mathbf{S}^{m-1}$; and

$$
M_{q}^{0,1}\left(\left\{c_{\mu}\right\}\right)=\sum_{\mu=1}^{\infty}\left|c_{\mu}\right|\left(1+\left(\log \frac{1}{\left|I_{\mu}\right|}\right)^{2}\right)<\infty .
$$

In dealing with singular integrals along subvarieties with rough kernels, an approach well-established by now is to decompose the operator into an infinite sum of Borel measures then to seek certain Fourier transform estimates and certain $L^{p}$ estimates of Littlewood-Paley type. For more details, we advise the readers to consult $[1,3,4,6,7$, $8,10]$, among others. A particular result that we will need to prove our results is the following result in [4] which is an extension of a result of Duoandikoetxea in [7].

TheOREM 2.4. Let $M, N \in \mathbb{N}$ and let $\left\{\sigma_{k, j}^{(l, s)}: k, j \in \mathbb{Z}, 0 \leq l \leq N, 0 \leq s \leq M\right\}$ be $a$ family of Borel measures on $\mathbb{R}^{n} \times \mathbb{R}^{m}$ with $\sigma_{k, j}^{(l, 0)}=0$ and $\sigma_{k, j}^{(0, s)}=0$ for every $k, j \in \mathbb{Z}$. Let $\left\{a_{l}, b_{s}: 1 \leq l \leq N, 1 \leq s \leq M\right\} \subseteq \mathbb{R}^{+} \backslash(0,2),\{B(l), D(s): 1 \leq l \leq N, 1 \leq s \leq M\} \subseteq \mathbb{N}$, $\left\{\alpha_{l}, \beta_{s}: 1 \leq l \leq N, 1 \leq s \leq M\right\} \subseteq \mathbb{R}^{+}$, and let $L_{l}: \mathbb{R}^{n} \rightarrow \mathbb{R}^{B(l)}$ and $Q_{s}: \mathbb{R}^{m} \rightarrow \mathbb{R}^{D(s)}$ be linear transformations for $1 \leq l \leq N, 1 \leq s \leq M$. Suppose that for some $B>1$ and $p_{0} \in(2, \infty)$ the following hold for $k, j \in \mathbb{Z}, 1 \leq l \leq N, 1 \leq s \leq M$, and $(\xi, \eta) \in \mathbb{R}^{n} \times \mathbb{R}^{m}$ :

(i) $\left\|\sigma_{k, j}^{(l, s)}\right\| \leq B^{2}$;

(ii) $\left|\hat{\sigma}_{k, j}^{(l, s)}(\xi, \eta)\right| \leq B^{2}\left|a_{l}^{k B} L_{l}(\xi)\right|^{-\alpha_{l} / B}\left|b_{s}^{j B} Q_{s}(\eta)\right|^{-\beta_{s} / B}$;

(iii) $\left|\hat{\sigma}_{k, j}^{(l, s)}(\xi, \eta)-\hat{\sigma}_{k, j}^{(l-1, s)}(\xi, \eta)\right| \leq B^{2}\left|a_{l}^{k B} L_{l}(\xi)\right|^{\alpha_{l} / B}\left|b_{s}^{j B} Q_{s}(\eta)\right|^{-\beta_{s} / B}$;

(iv) $\left|\hat{\sigma}_{k, j}^{(l, s)}(\xi, \eta)-\hat{\sigma}_{k, j}^{(l, s-1)}(\xi, \eta)\right| \leq B^{2}\left|a_{l}^{k B} L_{l}(\xi)\right|^{-\alpha_{l} / B}\left|b_{s}^{j B} Q_{s}(\eta)\right|^{\beta_{s} / B}$;

(v) $\left|\hat{\sigma}_{k, j}^{(l, s)}(\xi, \eta)-\hat{\sigma}_{k, j}^{(l-1, s)}(\xi, \eta)-\hat{\sigma}_{k, j}^{(l, s-1)}(\xi, \eta)+\hat{\sigma}_{k, j}^{(l-1, s-1)}(\xi, \eta)\right| \leq B^{2}\left|a_{l}^{k B} L_{l}(\xi)\right|^{\alpha_{l} / B} \times$ $\left|b_{s}^{j B} Q_{s}(\eta)\right|^{\beta_{s} / B}$

(vi) $\left|\hat{\sigma}_{k, j}^{(l, s-1)}(\xi, \eta)-\hat{\sigma}_{k, j}^{(l-1, s-1)}(\xi, \eta)\right| \leq B^{2}\left|a_{l}^{k B} L_{l}(\xi)\right|^{\alpha_{l} / B}$;

(vii) $\left|\hat{\sigma}_{k, j}^{(l-1, s)}(\xi, \eta)-\hat{\sigma}_{k, j}^{(l-1, s-1)}(\xi, \eta)\right| \leq B^{2}\left|b_{s}^{j B} Q_{s}(\eta)\right|^{\beta_{s} / B}$;

(viii) for arbitrary functions $g_{k, j}$ on $\mathbb{R}^{n} \times \mathbb{R}^{m}$,

$$
\left\|\left(\sum_{k, j \in \mathbb{Z}}\left|\sigma_{k, j}^{(l, s)} * g_{k, j}\right|^{2}\right)^{1 / 2}\right\|_{p_{0}} \leq B^{2}\left\|\left(\sum_{k, j \in \mathbb{Z}}\left|g_{k, j}\right|^{2}\right)^{1 / 2}\right\|_{p_{0}} .
$$

Then for $p_{0}^{\prime}<p<p_{0}$, where $p_{0}^{\prime}$ is the conjugate exponent of $p_{0}$, there exists a positive constant $C_{p}$ such that

$$
\begin{gathered}
\left\|\sum_{k, j \in \mathbb{Z}} \sigma_{k, j}^{(N, M)} * f\right\|_{L^{p}\left(\mathbb{R}^{n} \times \mathbb{R}^{m}\right)} \leq C_{p} B^{2}\|f\|_{L^{p}\left(\mathbb{R}^{n} \times \mathbb{R}^{m}\right)}, \\
\left\|\left(\sum_{k, j \in \mathbb{Z}}\left|\sigma_{k, j}^{(N, M)} * f\right|^{2}\right)^{(1 / 2)}\right\|_{L^{p}\left(\mathbb{R}^{n} \times \mathbb{R}^{m}\right)} \leq C_{p} B^{2}\|f\|_{L^{p}\left(\mathbb{R}^{n} \times \mathbb{R}^{m}\right)}
\end{gathered}
$$

hold for all $f$ in $L^{p}\left(\mathbb{R}^{n} \times \mathbb{R}^{m}\right)$. The constant $C_{p}$ is independent of the linear transformations $\left\{L_{l}\right\}_{l=1}^{N}$ and $\left\{Q_{s}\right\}_{s=1}^{M}$. 
It is clear that inequality (2.4) is one of the key elements in Theorem 2.4. In particular, the range of the parameter $p$ where (2.5) and (2.6) hold is completely determined by the largest $p_{0}$ where (2.4) holds. Clearly, if (2.4) holds for large $p_{0} \rightarrow \infty$, then (2.5) and (2.6) hold for all $1<p<\infty$. It turns out that to prove our results, we will indeed run into the case where we need to obtain (2.5) and (2.6) for all $1<p<\infty$. However, in our case this obstacle can be resolved. In fact, we will show that inequality (2.4) holds for all $p_{0}=4,8,16, \ldots$. Our main tools to achieve this are Lemma 2.5 and Theorem 2.6.

By a quick investigation of the proof of [7, Lemma 1], we have the following.

LEMMA 2.5. Let $\left\{v_{k, j}: k, j \in \mathbb{Z}\right\}$ be a sequence of Borel measures in $\mathbb{R}^{n} \times \mathbb{R}^{m}$ and let $v^{*}(f)=\sup _{k, j \in \mathbb{Z}}|| v_{k, j}|* f|$. Suppose that for some $q>1$ and $A>0$,

$$
\left\|v^{*}(f)\right\|_{q} \leq A\|f\|_{q}
$$

for every $f$ in $L^{q}\left(\mathbb{R}^{n} \times \mathbb{R}^{m}\right)$. Then the vector-valued inequality

$$
\left\|\left(\sum_{k, j \in \mathbb{Z}}\left|v_{k, j} * g_{k, j}\right|^{2}\right)^{1 / 2}\right\| \leq \sqrt{A \sup _{k, j \in \mathbb{Z}}\left\|v_{k, j}\right\|}\left\|\left(\sum_{p_{0}}\left|g_{k, j}\right|^{2}\right)^{1 / 2}\right\|_{p_{0}}
$$

holds for $\left|1 / p_{0}-1 / 2\right|=1 / 2 q$ and for arbitrary functions $\left\{g_{k, j}\right\}$ on $\mathbb{R}^{n} \times \mathbb{R}^{m}$.

Clearly, if inequality (2.7) holds for all $1<q<\infty$, then inequality (2.8) holds for all $p_{0}=4,8,16, \ldots$ which is the case that we will need to prove our results. But in many applications including the ones in this paper inequality (2.7) is not always freely available for all $1<q<\infty$. However, this problem can be resolved by repeated use of Theorem 2.4 and Lemma 2.5 along with a certain bootstrapping argument (see (2.15)(2.22)). To be more specific, we prove the following theorem.

THEOREM 2.6. Let $m, n, M, N \in \mathbb{N}, B>1, a, b \geq 2, \alpha, \beta>0$, and let $L: \mathbb{R}^{n} \rightarrow \mathbb{R}^{N}$ and $Q: \mathbb{R}^{m} \rightarrow \mathbb{R}^{M}$ be linear transformations. Let $\left\{\lambda_{k, j}^{(l, s)}: k, j \in \mathbb{Z}, l=1,2, s=1,2\right\}$ be a sequence of nonnegative Borel measures on $\mathbb{R}^{n} \times \mathbb{R}^{m}$ with $\left\|\lambda_{k, j}^{(l, s)}\right\| \leq B^{2}, 1 \leq l, s \leq 2$. Suppose that

(i) $\left|\hat{\lambda}_{k, j}^{(2,2)}(\xi, \eta)\right| \leq B^{2}\left|a^{k B} L(\xi)\right|^{-\alpha / B}\left|b^{j B} Q(\eta)\right|^{-\beta / B}$;

(ii) $\left|\hat{\lambda}_{k, j}^{(2,2)}(\xi, \eta)-\hat{\lambda}_{k, j}^{(1,2)}(\xi, \eta)\right| \leq B^{2}\left|a^{k B} L(\xi)\right|^{\alpha / B}\left|b^{j B} Q(\eta)\right|^{-\beta / B}$;

(iii) $\left|\hat{\lambda}_{k, j}^{(2,2)}(\xi, \eta)-\hat{\lambda}_{k, j}^{(2,1)}(\xi, \eta)\right| \leq B^{2}\left|a^{k B} L(\xi)\right|^{-\alpha / B}\left|b^{j B} Q(\eta)\right|^{\beta / B}$;

(iv) $\left|\hat{\lambda}_{k, j}^{(2,2)}(\xi, \eta)-\hat{\lambda}_{k, j}^{(1,2)}(\xi, \eta)-\hat{\lambda}_{k, j}^{(2,1)}(\xi, \eta)+\hat{\lambda}_{k, j}^{(1,1)}(\xi, \eta)\right| \leq B^{2}\left|a^{k B} L(\xi)\right|^{\alpha / B}\left|b^{j B} Q(\eta)\right|^{\beta / B}$;

(v) $\left|\hat{\lambda}_{k, j}^{(2,1)}(\xi, \eta)-\hat{\lambda}_{k, j}^{(1,1)}(\xi, \eta)\right| \leq B^{2}\left|a^{k B} L(\xi)\right|^{\alpha / B}$;

(vi) $\left|\hat{\lambda}_{k, j}^{(1,2)}(\xi, \eta)-\hat{\lambda}_{k, j}^{(1,1)}(\xi, \eta)\right| \leq B^{2}\left|b^{j B} Q(\eta)\right|^{\beta / B}$.

Suppose also that the maximal functions $M^{(l, s)}(f)=\sup _{k, j \in \mathbb{Z}}|| \lambda_{k, j}^{(l, s)}|* f|, 1 \leq l, s \leq 2$, satisfy

$$
\left\|M^{(l, s)}(f)\right\|_{p} \leq B^{2}\|f\|_{p}
$$

for all $(l, s) \in\{(1,2),(2,1),(1,1)\}, 1<p \leqslant \infty$, and $f \in L^{p}\left(\mathbb{R}^{n} \times \mathbb{R}^{m}\right)$. 
Then the inequality

$$
\left\|M^{(2,2)}(f)\right\|_{p} \leq C B^{2}\|f\|_{p}
$$

holds for all $1<p \leqslant \infty$, and $f$ in $L^{p}\left(\mathbb{R}^{n} \times \mathbb{R}^{m}\right)$. The constant $C$ is independent of $B$ and the linear transformations $L$ and $Q$.

Proof. Let $d$ be a fixed positive integer. For $1 \leq u \leq d$, we let $\pi_{u}^{d}: \mathbb{R}^{d} \rightarrow \mathbb{R}^{u}$ be the projection operator. By a similar argument to that in [10], we may assume that $N \leq n, M \leq m, L=\pi_{N}^{n}$, and $Q=\pi_{M}^{m}$. Choose and fix a Schwartz function $\phi \in \mathscr{Y}(\mathbb{R})$ such that $(\hat{\phi})(t) \equiv 1$ if $|t| \leq 1 / 2$ and $(\hat{\phi})(t) \equiv 0$ if $|t| \geq 1$. Define $\varphi_{k}$ on $\mathbb{R}^{N}$ and $\psi_{j}$ on $\mathbb{R}^{M}$ by $\left(\hat{\varphi}_{k}\right)(w)=(\hat{\phi})\left(\left|a^{k B} w\right|^{2}\right)$ and $\left(\hat{\psi}_{j}\right)(z)=(\hat{\phi})\left(\left|b^{j B} z\right|^{2}\right)$. Define the sequence of measures $\left\{\Gamma_{k, j}\right\}$ by

$$
\begin{aligned}
\hat{\Gamma}_{k, j}(\xi, \eta)= & \hat{\lambda}_{k, j}^{(2,2)}(\xi, \eta)-\hat{\lambda}_{k, j}^{(1,2)}(\xi, \eta)\left(\hat{\varphi}_{k}\right)\left(\pi_{N}^{n} \xi\right)-\hat{\lambda}_{k, j}^{(2,1)}(\xi, \eta) \\
& \times\left(\hat{\psi}_{j}\right)\left(\pi_{M}^{m} \eta\right)+\hat{\lambda}_{k, j}^{(1,1)}(\xi, \eta)\left(\hat{\varphi}_{k}\right)\left(\pi_{N}^{n} \xi\right)\left(\hat{\psi}_{j}\right)\left(\pi_{M}^{m} \eta\right) .
\end{aligned}
$$

Then one can easily verify that

$$
\left|\hat{\Gamma}_{k, j}(\xi, \eta)\right| \leq C B^{2}\left(a^{k B}|L(\xi)|\right)^{ \pm \alpha / 2 B}\left(b^{j B}|Q(\eta)|\right)^{ \pm \beta / 2 B}
$$

for $(\xi, \eta) \in \mathbb{R}^{n} \times \mathbb{R}^{m}$. Let $g(f)=\left(\sum_{k, j \in \mathbb{Z}}\left|\Gamma_{k, j} * f\right|^{2}\right)^{1 / 2}$ and $\Gamma^{*}(f)=\sup _{k, j \in \mathbb{Z}}|| \Gamma_{k, j}|* f|$. By (2.11) we have

$$
\begin{aligned}
M^{(2,2)} f(x, y) \leq & g(f)(x, y)+C\left(\left(\mu_{\mathbb{R}^{N}} \otimes i d_{\mathbb{R}^{n-N}}\right) \otimes i d_{\mathbb{R}^{M}}\right)\left(M^{(1,2)} f(x, y)\right) \\
& +C\left(i d_{\mathbb{R}^{N}} \otimes\left(\mu_{\mathbb{R}^{M}} \otimes i d_{\mathbb{R}^{m-M}}\right)\right)\left(M^{(2,1)} f(x, y)\right) \\
& +C\left(\left(\mu_{\mathbb{R}^{N}} \otimes i d_{\mathbb{R}^{n-N}}\right) \otimes\left(\mu_{\mathbb{R}^{M}} \otimes i d_{\mathbb{R}^{m-M}}\right)\right)\left(M^{(1,1)} f(x, y)\right), \\
\Gamma^{*} f(x, y) \leq & g(f)(x, y)+2 C\left(\left(\mu_{\mathbb{R}^{N}} \otimes i d_{\mathbb{R}^{n-N}}\right) \otimes i d_{\mathbb{R}^{M}}\right)\left(M^{(1,2)} f(x, y)\right) \\
& +2 C\left(i d_{\mathbb{R}^{N}} \otimes\left(\mu_{\mathbb{R}^{M}} \otimes i d_{\mathbb{R}^{m-M}}\right)\right)\left(M^{(2,1)} f(x, y)\right) \\
& +2 C\left(\left(\mu_{\mathbb{R}^{N}} \otimes i d_{\mathbb{R}^{n-N}}\right) \otimes\left(\mu_{\mathbb{R}^{M}} \otimes i d_{\mathbb{R}^{m-M}}\right)\right)\left(M^{(1,1)} f(x, y)\right),
\end{aligned}
$$

where $\mathcal{M}_{\mathbb{R}^{d}}$ is the classical Hardy-Littlewood maximal function on $\mathbb{R}^{d}$.

By Plancherel's theorem and (2.12), we get

$$
\|g(f)\|_{L^{2}} \leq C B^{2}\|f\|_{L^{2}}
$$

which implies by (2.9) and (2.14) that

$$
\left\|\Gamma^{*}(f)\right\|_{L^{2}} \leq C B^{2}\|f\|_{L^{2}}
$$

By applying Lemma 2.5 (for $q=2$ ) along with the trivial estimate $\left\|\Gamma_{k, j}\right\| \leq C B^{2}$, we get

$$
\left\|\left(\sum_{k, j \in \mathbb{Z}}\left|\Gamma_{k, j} * g_{k, j}\right|^{2}\right)^{1 / 2}\right\|_{p_{0}} \leq C_{p_{0}} B^{2}\left\|\left(\sum_{k, j \in \mathbb{Z}}\left|g_{k, j}\right|^{2}\right)^{1 / 2}\right\|_{p_{0}}
$$


for all $p_{0}$ satisfying $1 / 4=\left|1 / p_{0}-1 / 2\right|$. By Theorem 2.4 , (2.12), and (2.17), we obtain

$$
\|g(f)\|_{L^{p}} \leq C_{p} B^{2}\|f\|_{L^{p}} \quad \text { for } \frac{4}{3}<p<4
$$

which implies by (2.9) and (2.14) that

$$
\left\|\Gamma^{*}(f)\right\|_{L^{p}} \leq C B^{2}\|f\|_{L^{p}} \quad \text { for } \frac{4}{3}<p<4
$$

Reasoning as above, we get

$$
\|g(f)\|_{L^{p}} \leq C_{p} B^{2}\|f\|_{L^{p}} \text { for } \frac{8}{7}<p<8 .
$$

By repeating the above argument we eventually get

$$
\|g(f)\|_{L^{p}} \leq C_{p} B^{2}\|f\|_{L^{p}} \quad \text { for } 1<p<\infty
$$

which when combined with (2.9) and (2.13) implies that

$$
\left\|M^{(2,2)}(f)\right\|_{L^{p}} \leq C_{p} B^{2}\|f\|_{L^{p}} \text { for } 1<p<\infty .
$$

For $p=\infty$, the inequality holds trivially. The proof of the theorem is complete.

For $\rho \geq 2, k, j \in \mathbb{Z}_{-}$, let $D(k, j, \rho)=\left\{(u, v) \in \mathbb{R}^{n} \times \mathbb{R}^{m}: \rho^{k-1} \leq|u|<\rho^{k}, \rho^{j-1} \leq|v|<\right.$ $\left.\rho^{j}\right\}$. For suitable mappings $\Gamma: \mathbb{R}^{n} \rightarrow \mathbb{R}^{N}, \Lambda: \mathbb{R}^{m} \rightarrow \mathbb{R}^{M}$, and $\tilde{b}: \mathbf{S}^{n-1} \times \mathbf{S}^{m-1} \rightarrow \mathbb{R}$, we define the measures $\left\{\Delta_{\tilde{b}, \Gamma, \Lambda, k, j, \rho}: k, j \in \mathbb{Z}_{-}\right\}$and the related maximal operator $\Delta_{\tilde{b}, \Gamma, \Lambda, \rho}^{*}$ on $\mathbb{R}^{N} \times \mathbb{R}^{M}$ by

$$
\begin{gathered}
\iint_{\mathbb{R}^{N} \times \mathbb{R}^{M}} f d \Delta_{\tilde{b}, \Gamma, \Lambda, k, j, \rho}=\iint_{D(k, j, \rho)} f(\Gamma(x), \Lambda(y)) \tilde{b}\left(x^{\prime}, y^{\prime}\right)|x|^{-n}|y|^{-m} d x d y, \\
\Delta_{\tilde{b}, \Gamma, \Lambda, \rho}^{*} f(x, y)=\sup _{k, j \in \mathbb{Z}-}|| \Delta_{\tilde{b}, \Gamma, \Lambda, k, j, \rho}|* f(x, y)| .
\end{gathered}
$$

For $l \in \mathbb{N}$, let $\mathscr{A}_{l}$ denote the class of polynomials of $l$ variables with real coefficients. For $d \in \mathbb{N}$ and $\mathscr{R}=\left(\mathscr{R}_{1}, \ldots, \mathscr{R}_{d}\right) \in\left(\mathscr{A}_{1}\right)^{d}$, define the maximal function $M_{\mathscr{R}} f$ on $\mathbb{R}^{d}$ by

$$
M_{\Re} f(x)=\sup _{r>0} \frac{1}{r} \int_{-r}^{r}|f(x-\mathscr{R}(t))| d t .
$$

The following result can be found in [15].

LEMMA 2.7. For $1<p \leq \infty$, there exists a positive constant $C_{p}$ such that

$$
\left\|M_{\Re} f\right\|_{p} \leq C_{p}\|f\|_{p}
$$

for $f \in L^{p}\left(\mathbb{R}^{d}\right)$. The constant $C_{p}$ may depend on the degrees of the polynomials $\mathscr{R}_{1}, \ldots$, $\Re_{d}$, but it is independent of their coefficients. 
LEMMA 2.8. Let $\Phi: B_{n}(0,1) \rightarrow \mathbb{R}^{N}$ and $\Psi: B_{m}(0,1) \rightarrow \mathbb{R}^{M}$ be $C^{\infty}$ mappings and let, $\mathscr{P}=\left(P_{1}, \ldots, P_{N}\right): \mathbb{R}^{n} \rightarrow \mathbb{R}^{N}$ and $\mathscr{2}=\left(Q_{1}, \ldots, Q_{M}\right): \mathbb{R}^{m} \rightarrow \mathbb{R}^{M}$ be polynomial mappings. Let $\tilde{b}(\cdot, \cdot)$ be a function on $\mathbf{S}^{n-1} \times \mathbf{S}^{m-1}$ satisfying the following conditions:

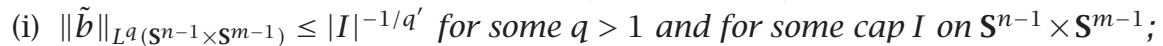

(ii) $\|\tilde{b}\|_{L^{1}\left(S^{n-1} \times S^{m-1}\right)} \leq 1$. Let $\rho=2^{[\log (1 /|I|)]}$ and $B(I, \rho)=\log (1 /|I|)$ if $|I|<e^{-1}$ and let $\rho=2$ and $B(I, \rho)=1$ if $|I| \geq e^{-1}$, where $[\cdot]$ is the greatest integer function.

If $\Phi$ and $\Psi$ are of finite type at 0 , then for $1<p \leq \infty$ and $f \in L^{p}\left(\mathbb{R}^{N} \times \mathbb{R}^{M}\right)$ there exists a positive constant $C_{p}$ which is independent of $\tilde{b}$ such that

$$
\begin{aligned}
&\left\|\Delta_{\tilde{b}, \mathscr{P}, \Psi, \rho}^{*}(f)\right\|_{L^{p}\left(\mathbb{R}^{N} \times \mathbb{R}^{M}\right)} \leq C_{p}[B(I, \rho)]^{2}\|f\|_{L^{p}\left(\mathbb{R}^{N} \times \mathbb{R}^{M}\right)}, \\
&\left\|\Delta_{\tilde{b}, \Phi, \mathscr{Q}, \rho}^{*}(f)\right\|_{L_{\left(\mathbb{R}^{N} \times \mathbb{R}^{M}\right)}} \leq C_{p}[B(I, \rho)]^{2}\|f\|_{L_{\left(\mathbb{R}^{N} \times \mathbb{R}^{M}\right)}} .
\end{aligned}
$$

Proof. We will only present the proof of (2.26). By the definition of $\Delta_{\tilde{b}, \mathscr{P}, \Psi, \rho}^{*}$ we notice that $\Delta_{\tilde{b}, \mathscr{P}, \Psi, \rho}^{*} f(x, y)$ is dominated by

$$
\sup _{j \in \mathbb{Z}_{-}} \int_{\rho^{j-1} \leq|v|<\rho^{j}} \frac{1}{|v|^{m}} \int_{S^{n-1}}|\tilde{b}(u, v)|\left|\left(M_{\mathscr{P}, \rho, u} f(\cdot, y-\Psi(v))\right)(x)\right| d \sigma(u) d v,
$$

where

$$
\mu_{\mathscr{P}, \rho, u} h(x)=\sup _{k \in \mathbb{Z}_{-}} \int_{\rho^{k-1}}^{\rho^{k}}|h(x-\mathscr{P}(t u))| \frac{d t}{t} .
$$

By Lemma 2.7 we immediately get

$$
\left\|\Delta_{\tilde{b}, \mathscr{P}, \Psi, \rho}^{*}(f)\right\|_{L^{p}\left(\mathbb{R}^{N} \times \mathbb{R}^{M}\right)} \leq C_{p}[B(I, \rho)]\left(\int_{\mathbb{R}^{M}}\left\|\mathscr{H}_{\Psi, \tilde{b}_{0}} f(\cdot, y)\right\|_{L^{p}\left(\mathbb{R}^{N}\right)}^{p} d y\right)^{1 / p},
$$

where

$$
\mathscr{H}_{\Psi, \tilde{b}_{0}} g(y)=\sup _{j \in \mathbb{Z}-} \int_{\rho^{j-1} \leq|v|<\rho^{j}}|g(y-\Psi(v))| \frac{\tilde{b}_{0}(v)}{|v|^{m}} d v
$$

and $\tilde{b}_{0}$ is a function on $\mathbf{S}^{m-1}$ defined by $\tilde{b}_{0}(v)=\int_{\mathbf{S}^{n-1}}|\tilde{b}(u, v)| d \sigma(u)$. By the arguments in the proof of the $L^{p}$ boundedness of the corresponding maximal function in the oneparameter setting in [1, Theorem 3.8], we obtain (2.26). This ends the proof of our theorem.

By Lemma 2.7 we immediately get the following.

LEMMA 2.9. Let $\mathscr{P}=\left(P_{1}, \ldots, P_{N}\right): \mathbb{R}^{n} \rightarrow \mathbb{R}^{N}$ and $\mathscr{2}=\left(Q_{1}, \ldots, Q_{M}\right): \mathbb{R}^{m} \rightarrow \mathbb{R}^{M}$ be polynomial mappings. Let $\tilde{b}(\cdot, \cdot)$ be as in Lemma 2.8. Then for $1<p \leq \infty$, there exists a constant $C_{p}$ such that

$$
\left\|\Delta_{\tilde{b}, \mathscr{P}, \mathscr{Q}, \rho}^{*}(f)\right\|_{p} \leq C_{p}[B(I, \rho)]^{2}\|f\|_{L^{p}\left(\mathbb{R}^{N} \times \mathbb{R}^{M}\right)}
$$

for $f \in L^{p}\left(\mathbb{R}^{N} \times \mathbb{R}^{M}\right)$. 
3. Certain Fourier transform estimates. We will need the following two lemmas from [8].

LEMMA 3.1. Let $\Phi: B(0,1) \rightarrow \mathbb{R}^{d}$ be a smooth mapping and let $\Omega$ be a homogeneous function on $\mathbb{R}^{n}$ of degree 0 . Suppose that $\Phi$ is of finite type at 0 and $\Omega \in L^{q}\left(\mathbf{S}^{n-1}\right)$ for some $q>1$. Then there are $N_{0} \in \mathbb{N}, \delta \in(0,1], C>0$, and $j_{0} \in \mathbb{Z}_{-}$such that

$$
\left|\int_{2^{j-1} \leq|y|<2^{j}} e^{-i \xi \cdot \Phi(y)} \frac{\Omega(y)}{|y|^{n}} d y\right| \leq C\|\Omega\|_{L^{q\left(\mathbf{S}^{n-1}\right)}}\left(2^{N_{0} j}|\xi|\right)^{-\delta}
$$

for all $j \leq j_{0}$ and $\xi \in \mathbb{R}^{d}$.

LEMmA 3.2. Let $m \in \mathbb{N}$ and let $R(\cdot)$ be a real-valued polynomial on $\mathbb{R}^{n}$ with $\operatorname{deg}(R) \leqslant$ $m-1$. Suppose that $P(y)=\sum_{|\alpha|=m} a_{\alpha} y^{\alpha}+R(y), \Omega$ is a homogeneous function of degree zero, and $\Omega \in L^{q}\left(\mathbf{S}^{n-1}\right)$ for some $q>1$. Then there exists a constant $C>0$ such that

$$
\left|\int_{2^{j-1} \leq|y|<2^{j}} e^{-i P(y)} \frac{\Omega(y)}{|y|^{n}} d y\right| \leq C\|\Omega\|_{L^{q\left(\mathbf{S}^{n-1}\right)}}\left(2^{m j} \sum_{|\alpha|=m}\left|a_{\alpha}\right|\right)^{-1 / 2 q^{\prime} m}
$$

holds for all $j \in \mathbb{Z}_{-}$and $a_{\alpha} \in \mathbb{R}$.

LEMMA 3.3. Let $\Phi: B_{n}(0,1) \rightarrow \mathbb{R}^{N}$ and $\Psi: B_{m}(0,1) \rightarrow \mathbb{R}^{M}$ be $C^{\infty}$ mappings and let $\tilde{b}(\cdot, \cdot), I, \rho$, and $B(I, \rho)$ be as in Lemma 2.8. Suppose that $\Phi$ and $\Psi$ are of finite type at 0 . Then there are $N_{0}, M_{0} \in \mathbb{N}, \delta \in(0,1], C>0$, and $j_{0}, k_{0} \in \mathbb{Z}_{-}$such that

$$
\left|\hat{\Delta}_{\tilde{b}, \Phi, \Psi, k, j, \rho}(\xi, \eta)\right| \leq C[B(I, \rho)]^{2}\left(\rho^{N_{0} k}|\xi|\right)^{-\delta / B(I, \rho)}\left(\rho^{M_{0} j}|\eta|\right)^{-\delta / B(I, \rho)}
$$

for all $k \leq k_{0}, j \leq j_{0}$, and $(\xi, \eta) \in \mathbb{R}^{N} \times \mathbb{R}^{M}$.

Proof. We start by the proof of (3.3) for the case $|I|<e^{-1}$. By the definition of $\Delta_{\tilde{b}, \Phi, \Psi, k, j, \rho}$, we get

$$
\left|\hat{\Delta}_{\tilde{b}, \Phi, \Psi, k, j, \rho}(\xi, \eta)\right| \leq C\left[\log \left(\frac{1}{|I|}\right)\right] \int_{\mathbf{S}^{m-1}} S_{k}(y, \xi) d \sigma(y),
$$

where

$$
S_{k}(y, \xi)=\left|\int_{\rho^{k-1} \leq|x|<\rho^{k}} e^{-i \xi \cdot \Phi(x)} \frac{\tilde{b}(x, y)}{|x|^{n}} d x\right| .
$$

Now, by Lemma 3.1,

$$
\begin{aligned}
\left|S_{k}(y, \xi)\right| & \leq \sum_{s=0}^{[\log 1 /|I|]-1} \int_{\mathbf{S}^{m-1}}\left|\int_{\rho^{(k-1)} 2^{s} \leq|x|<\rho^{(k-1)} 2^{(s+1)}} e^{-i \xi \cdot \Phi(x)} \frac{\tilde{b}(x, y)}{|x|^{n}} d x\right| \\
& \leq C \sum_{s=0}^{[\log 1 /|I|]-1} \int_{\mathbf{S}^{m-1}}\|\tilde{b}(\cdot, y)\|_{L^{q}\left(\mathbf{S}^{n-1)}\right.}\left(\rho^{N_{0}(k-1)} 2^{N_{0}(s+1)}|\xi|\right)^{-\delta} \\
& \leq C \rho^{\delta N_{0}} \int_{\mathbf{S}^{m-1}}\|\tilde{b}(\cdot, y)\|_{L^{q\left(\mathbf{S}^{n-1}\right)}}\left(\rho^{N_{0} k}|\xi|\right)^{-\delta} .
\end{aligned}
$$


Therefore, by Lemma 2.8(i) and Hölder's inequality, we have

$$
\left|\hat{\Delta}_{\tilde{b}, \Phi, \Psi, k, j, \rho}(\xi, \eta)\right| \leq C \rho^{\delta N}|I|^{-1 / q^{\prime}}\left(\rho^{N_{0} k}|\xi|\right)^{-\delta}
$$

which, when combined with the trivial bound $\left|\hat{\mho}_{\tilde{b}, \Phi, \Psi, k, j, \rho}(\xi, \eta)\right| \leq C[\log (1 /|I|)]^{2}$, implies

$$
\left|\hat{\Delta}_{\tilde{b}, \Phi, \Psi, k, j, \rho}(\xi, \eta)\right| \leq C\left[\log \left(\frac{1}{|I|}\right)\right]^{2}\left(\rho^{N_{0} k}|\xi|\right)^{-\delta /[\log (1 /|I|)]}
$$

Similarly, we have

$$
\left|\hat{\Delta}_{\tilde{b}, \Phi, \Psi, k, j, \rho}(\xi, \eta)\right| \leq C\left[\log \left(\frac{1}{|I|}\right)\right]^{2}\left(\rho^{M_{0} j}|\eta|\right)^{-\delta /[\log (1 /|I|)]} .
$$

Combining estimates (3.8) and (3.9) yields the estimate in (3.3) when $|I|<e^{-1}$.

The proof of (3.3) for the case $|I| \geq e^{-1}$ follows by exactly the same argument as that for the case $|I|<e^{-1}$ but this time we replace $\rho$ and $\log (1 /|I|)$ by 2 and $\log (2)$, respectively, and use the observation that $|I|^{-1 / q^{\prime}} \leq e$. This concludes the proof of our lemma.

By Lemma 3.2 and the same argument employed in the proof of Lemma 3.3, we get the following.

LEMMA 3.4. Let $N_{0}, M_{0} \in \mathbb{N}$, and let $\tilde{b}(\cdot, \cdot), I, \rho$, and $B(I, \rho)$ be as in Lemma 2.8. Let $R_{1}(\cdot)$ and $R_{2}(\cdot)$ be real-valued polynomials on $\mathbb{R}^{n}$ and $\mathbb{R}^{m}$, respectively, with $\operatorname{deg}\left(R_{1}\right) \leqslant$ $N_{0}-1$ and $\operatorname{deg}\left(R_{2}\right) \leqslant M_{0}-1$. Let $P(x)=\sum_{|\alpha|=N_{0}} a_{\alpha} x^{\alpha}+R_{1}(x)$ and $Q(y)=\sum_{|\beta|=M_{0}} b_{\beta} y^{\beta}+$ $R_{2}(y)$. Then there exists a constant $C>0$ such that for all $k, j \in \mathbb{Z}$ and $a_{\alpha}, b_{\beta} \in \mathbb{R}$,

$$
\begin{aligned}
& \left|\iint_{D(k, j, \rho)} e^{i(P(x)+Q(y))} \frac{\tilde{b}(x, y)}{|x|^{n}|y|^{m}} d x d y\right| \\
& \quad \leq C[B(I, \rho)]^{2}\left(\rho^{N_{0} k} \sum_{|\alpha|=N_{0}}\left|a_{\alpha}\right|\right)^{-1 / 2 \dot{q} N_{0} B(I, \rho)}\left(\rho^{M_{0} j} \sum_{|\beta|=M_{0}}\left|b_{\beta}\right|\right)^{-1 / 2 \dot{q} M_{0} B(I, \rho)} .
\end{aligned}
$$

THEOREM 3.5. Let $\Phi: B_{n}(0,1) \rightarrow \mathbb{R}^{N}$ and $\Psi: B_{m}(0,1) \rightarrow \mathbb{R}^{M}$ be $C^{\infty}$ mappings and let $\tilde{b}(\cdot, \cdot), I, \rho$, and $B(I, \rho)$ be as in Lemma 2.8. Suppose that $\Phi$ and $\Psi$ are of finite type at 0 . Then for $1<p \leq \infty$ and $f \in L^{p}\left(\mathbb{R}^{N} \times \mathbb{R}^{M}\right)$, there exists a positive constant $C_{p}$ which is independent of $\tilde{b}$ such that

$$
\left\|\Delta_{\tilde{b}, \Phi, \Psi, \rho}^{*}(f)\right\|_{p} \leq C_{p}[B(I, \rho)]^{2}\|f\|_{p} .
$$


Proof. Without loss of generality, we may assume that $\tilde{b} \geq 0$. Let $N_{0}, M_{0} \in \mathbb{N}$, $\delta \in(0,1], C>0$, and $k_{0}, j_{0} \in \mathbb{Z}_{-}$be as in Lemma 3.3. For $\Phi=\left(\Phi_{1}, \ldots, \Phi_{N}\right)$ and $\Psi=$ $\left(\Psi_{1}, \ldots, \Psi_{M}\right)$, we let $\mathscr{P}=\left(P_{1}, \ldots, P_{N}\right)$ and $\mathscr{2}=\left(Q_{1}, \ldots, Q_{M}\right)$, where

$$
\begin{gathered}
P_{l}(x)=\sum_{|\alpha|=N_{0}-1} \frac{1}{\alpha !} \frac{\partial^{\alpha} \Phi_{l}}{\partial x^{\alpha}}(0) x^{\alpha}, \\
Q_{s}(y)=\sum_{|\beta| \leqslant M_{0}-1} \frac{1}{\beta !} \frac{\partial^{\beta} \Psi_{s}}{\partial y^{\beta}}(0) y^{\beta},
\end{gathered}
$$

for $1 \leqslant s \leqslant M$ and $1 \leqslant l \leqslant N$. Then,

$$
\left|\hat{\Delta}_{\tilde{b}, \Phi, \Psi, k, j, \rho}(\xi, \eta)-\hat{\Delta}_{\tilde{b}, \Phi, \Psi, k, j, \rho}(\xi, \eta)\right| \leq C\left(\rho^{N_{0} k}|\xi|\right) \int_{S^{n-1}} H_{j}(x, \eta) d \sigma(x),
$$

where

$$
H_{j}(x, \eta)=\left|\int_{\rho^{j-1} \leq|y|<\rho^{j}} e^{-i \eta \cdot \Psi(y)} \frac{\tilde{b}(x, y)}{|y|^{m}} d y\right|
$$

By Lemma 3.1 and the argument in the proof of (3.3), we get

$$
\begin{aligned}
& \left|\hat{\Delta}_{\tilde{b}, \Phi, \Psi, k, j, \rho}(\xi, \eta)-\hat{\Delta}_{\tilde{b}, \mathscr{P}, \Psi, k, j, \rho}(\xi, \eta)\right| \\
& \quad \leq C[B(I, \rho)]^{2}\left(\rho^{N_{0} k}|\xi|\right)^{\delta /[B(I, \rho)]}\left(\rho^{M_{0} j}|\eta|\right)^{-\delta /[B(I, \rho)]} .
\end{aligned}
$$

Similarly, it is easy to verify that the following estimates hold:

$$
\begin{aligned}
& \left|\hat{\Delta}_{\tilde{b}, \Phi, \Psi, k, j, \rho}(\xi, \eta)-\hat{\Delta}_{\tilde{b}, \Phi, 2, k, j, \rho}(\xi, \eta)\right| \\
& \leq C[B(I, \rho)]^{2}\left(\rho^{N_{0} k}|\xi|\right)^{-\delta /[B(I, \rho)]}\left(\rho^{M_{0} j}|\eta|\right)^{\delta /[B(I, \rho)]}, \\
& \left|\hat{\Delta}_{\tilde{b}, \Phi, \Psi, k, j, \rho}(\xi, \eta)-\hat{\Delta}_{\tilde{b}, \Phi, \Psi, k, j, \rho}(\xi, \eta)-\hat{\Delta}_{\tilde{b}, \Phi, 2, k, j, \rho}(\xi, \eta)+\hat{\Delta}_{\tilde{b}, \Phi, Q, k, j, \rho}(\xi, \eta)\right| \\
& \leq C[B(I, \rho)]^{2}\left(\rho^{N_{0} k}|\xi|\right)^{\delta /[B(I, \rho)]}\left(\rho^{M_{0} j}|\eta|\right)^{\delta /[B(I, \rho)]}, \\
& \left|\hat{\Delta}_{\tilde{b}, \Phi, 2, k, j, \rho}(\xi, \eta)-\hat{\Delta}_{\tilde{b}, \mathscr{P}, \mathscr{Q}, k, j, \rho}(\xi, \eta)\right| \leq C[B(I, \rho)]^{2}\left(\rho^{N_{0} k}|\xi|\right)^{\delta /[B(I, \rho)]}, \\
& \left|\hat{\Delta}_{\tilde{b}, \mathscr{P}, \Psi, k, j, \rho}(\xi, \eta)-\hat{\Delta}_{\tilde{b}, \mathscr{P}, \mathscr{Q}, k, j, \rho}(\xi, \eta)\right| \leq C[B(I, \rho)]^{2}\left(\rho^{M_{0} j}|\eta|\right)^{\delta /[B(I, \rho)]} .
\end{aligned}
$$

By (3.3), (3.15)-(3.16), Theorem 2.6, and Lemmas 2.8 and 2.9, we get (3.11). This concludes the proof of the theorem.

4. Proof of Theorem 1.3. By assumption, $\Omega$ can be written as $\Omega=\sum_{\mu=1}^{\infty} c_{\mu} b_{\mu}$, where $c_{\mu} \in \mathbb{C}, b_{\mu}$ is $q$-block supported on a cap $I_{\mu}$ on $\mathbf{S}^{n-1} \times \mathbf{S}^{m-1}$, and $M_{q}^{0,1}\left(\left\{c_{\mu}\right\}\right)$ satisfies 
(2.3). Every $q$-block function $b_{\mu}(\cdot, \cdot)$ has a companion function $\tilde{b}_{\mu}(\cdot, \cdot)$ defined by

$$
\begin{aligned}
\tilde{b}_{\mu}(x, y)= & b_{\mu}(x, y)-\int_{\mathbf{S}^{n-1}} b_{\mu}(u, y) d \sigma(u)-\int_{\mathbf{S}^{m-1}} b_{\mu}(x, v) d \sigma(v) \\
& +\iint_{\mathbf{S}^{n-1} \times \mathbf{S}^{m-1}} b_{\mu}(u, v) d \sigma(u) d \sigma(v) .
\end{aligned}
$$

It is easy to verify that each $\tilde{b}_{\mu}$ enjoys the following properties:

$$
\begin{gathered}
\int_{\mathbf{S}^{n-1}} \tilde{b}_{\mu}(u, \cdot) d \sigma(u)=\int_{\mathbf{S}^{m-1}} \tilde{b}_{\mu}(\cdot, v) d \sigma(v)=0 ; \\
\left\|\tilde{b}_{\mu}\right\|_{L^{q\left(\mathbf{S}^{n-1} \times \mathbf{S}^{m-1}\right)} \leq 4|I|^{-1 / q^{\prime}} ; \quad\left\|\tilde{b}_{\mu}\right\|_{L^{1}\left(\mathbf{S}^{n-1} \times \mathbf{S}^{m-1}\right)} \leq 4 .}
\end{gathered}
$$

By the vanishing property on $\Omega$ we have

$$
\Omega=\sum_{\mu=1}^{\infty} c_{\mu} \tilde{b}_{\mu}
$$

and this yields

$$
\left\|P_{\Omega, \Phi, \Psi} f\right\|_{p} \leqslant \sum_{\mu=1}^{\infty}\left|c_{\mu}\right|\left\|T_{\tilde{b}_{\mu}} f\right\|_{p}
$$

where

$$
T_{\tilde{b}_{\mu}} f(x, y)=\text { p.v. } \iint_{B_{n}(0,1) \times B_{m}(0,1)} f(x-\Phi(u), y-\Psi(v)) \frac{\tilde{b}_{\mu}(u, v)}{|u|^{n}|v|^{m}} d u d v
$$

Let $N_{0}, M_{0}, \mathscr{P}$, and 2 be given as in the proof of Theorem 3.5. For $1 \leqslant l \leqslant N, 1 \leqslant s \leqslant M$, let $a_{l, \alpha}=(1 / \alpha !)\left(\partial^{\alpha} \Phi_{l} / \partial x^{\alpha}\right)(0)$ and $b_{s, \beta}=(1 / \beta !)\left(\partial^{\beta} \Psi_{s} / \partial y^{\beta}\right)(0)$. For $0 \leqslant \tau \leqslant N_{0}, 0 \leqslant \kappa \leqslant$ $M_{0}$, we define $P^{T}=\left(P_{l}^{T}, \ldots, P_{N}^{T}\right)$ and $Q^{K}=\left(Q_{1}^{K}, \ldots, Q_{M}^{K}\right)$ by

$$
\begin{aligned}
P_{l}^{\tau}(x) & =\sum_{|\alpha| \leqslant \tau} a_{l, \alpha} x^{\alpha}, \quad \text { for } l=1, \ldots, N, 0 \leqslant \tau \leqslant N_{0}-1 ; \\
Q_{S}^{\kappa}(y) & =\sum_{|\beta| \leqslant \kappa} b_{s, \beta} y^{\beta}, \quad \text { for } s=1, \ldots, M, 0 \leqslant \kappa \leqslant M_{0}-1 ;
\end{aligned}
$$

$P^{N_{0}}=\Phi$ and $Q^{M_{0}}=\Psi$. For each $\mu$, let $\rho_{\mu}$ and $B\left(I_{\mu}, \rho_{\mu}\right)$ be given by the same formulas for $\rho$ and $B(I, \rho)$ in Lemma 2.8 with $\rho, I$, and $B(I, \rho)$ replaced by $\rho_{\mu}, I_{\mu}$, and $B\left(I_{\mu}, \rho_{\mu}\right)$, respectively. For each $0 \leqslant \tau \leqslant N_{0}, 0 \leqslant \kappa \leqslant M_{0}$, let $\Delta_{\tilde{b}_{\mu}, k, j, \rho_{\mu}}^{(\tau, \kappa)}=\Delta_{\tilde{b}_{\mu}, P^{\tau}, Q^{\kappa}, k, j, \rho_{\mu}}$. Then by 
Lemma 3.4 and the same argument as in the proofs of (3.3) and (3.15), we get

$$
\begin{aligned}
& \left\|\Delta_{\tilde{b}_{\mu}, k, j, \rho_{\mu}}^{(\tau, \kappa)}\right\| \leqslant C\left[B\left(I_{\mu}, \rho_{\mu}\right)\right]^{2} ; \\
& \left|\hat{\Delta}_{\tilde{b}_{\mu}, k, j, \rho_{\mu}}^{(\tau, \kappa)}(\xi, \eta)\right| \leqslant C\left[B\left(I_{\mu}, \rho_{\mu}\right)\right]^{2}\left\{\rho_{\mu}^{\tau k} \sum_{|\alpha|=\tau}\left|\sum_{l=\tau}^{N} a_{l, \alpha} \xi_{l}\right|\right\}^{-\alpha_{\tau} /\left[B\left(I_{\mu}, \rho_{\mu}\right)\right]} \\
& \times\left[\rho_{\mu}^{\kappa j} \sum_{|\beta|=\kappa}\left|\sum_{s=\kappa}^{M} b_{s, \beta} \eta_{s}\right|\right]^{-\alpha_{\kappa}\left[B\left(I_{\mu}, \rho_{\mu}\right)\right]} ; \\
& \left|\hat{\Delta}_{\tilde{b}_{\mu}, k, j, \rho_{\mu}}^{(\tau, \kappa)}(\xi, \eta)-\hat{\Delta}_{\tilde{b}_{\mu}, k, j, \rho_{\mu}}^{(\tau-1, \kappa)}(\xi, \eta)\right| \leqslant C\left[B\left(I_{\mu}, \rho_{\mu}\right)\right]^{2}\left[\rho_{\mu}^{\tau k} \sum_{|\alpha|=\tau}\left|\sum_{l=\tau}^{N} a_{l, \alpha} \xi_{l}\right|\right]^{\alpha_{\tau}\left[B\left(I_{\mu}, \rho_{\mu}\right)\right]} \\
& \times\left[\rho_{\mu}^{\kappa j} \sum_{|\beta|=\kappa}\left|\sum_{s=\kappa}^{M} b_{s, \beta} \eta_{s}\right|\right]^{-\alpha_{\kappa} /\left[B\left(I_{\mu}, \rho_{\mu}\right)\right]} ; \\
& \left|\hat{\Delta}_{\tilde{b}_{\mu}, k, j, \rho_{\mu}}^{(\tau, \kappa)}(\xi, \eta)-\hat{\Delta}_{\tilde{b}_{\mu}, k, j, \rho_{\mu}}^{(\tau, \kappa-1)}(\xi, \eta)\right| \leqslant C\left[B\left(I_{\mu}, \rho_{\mu}\right)\right]^{2}\left[\rho_{\mu}^{\tau k} \sum_{|\alpha|=\tau}\left|\sum_{l=\tau}^{N} a_{l, \alpha} \xi_{l}\right|\right]^{-\alpha_{\tau} /\left[B\left(I_{\mu}, \rho_{\mu}\right)\right]} \\
& \times\left[\rho_{\mu}^{\kappa j} \sum_{|\beta|=\kappa}\left|\sum_{s=\kappa}^{M} b_{s, \beta} \eta_{s}\right|\right]^{\alpha_{K} /\left[B\left(I_{\mu}, \rho_{\mu}\right)\right]} ; \\
& \left|\hat{\Delta}_{\tilde{b}_{\mu}, k, j, \rho_{\mu}}^{(\tau, \kappa)}(\xi, \eta)-\hat{\Delta}_{\tilde{b}_{\mu}, k, j, \rho_{\mu}}^{(\tau-1, \kappa)}(\xi, \eta)-\hat{\Delta}_{\tilde{b}_{\mu}, k, j, \rho_{\mu}}^{(\tau, \kappa-1)}(\xi, \eta)+\hat{\Delta}_{\tilde{b}_{\mu}, k, j, \rho_{\mu}}^{(\tau-1, \kappa-1)}(\xi, \eta)\right| \\
& \leqslant C\left[B\left(I_{\mu}, \rho_{\mu}\right)\right]^{2}\left[\rho_{\mu}^{\tau k} \sum_{|\alpha|=\tau}\left|\sum_{l=\tau}^{N} a_{l, \alpha} \xi_{l}\right|\right]^{-\alpha_{\tau} /\left[B\left(I_{\mu}, \rho_{\mu}\right)\right]} \\
& \times\left[\rho_{\mu}^{\kappa j} \sum_{|\beta|=\kappa}\left|\sum_{s=\kappa}^{M} b_{s, \beta} \eta_{s}\right|\right]^{\alpha_{\kappa} /\left[B\left(I_{\mu}, \rho_{\mu}\right)\right]} ; \\
& \left|\hat{\Delta}_{\tilde{b}_{\mu}, k, j, \rho_{\mu}}^{(\tau, \kappa-1)}(\xi, \eta)-\hat{\Delta}_{\tilde{b}_{\mu}, k, j, \rho_{\mu}}^{(\tau-1, \kappa-1)}(\xi, \eta)\right| \leqslant C\left[B\left(I_{\mu}, \rho_{\mu}\right)\right]^{2}\left[\rho_{\mu}^{\tau k} \sum_{|\alpha|=\tau}\left|\sum_{l=\tau}^{N} a_{l, \alpha} \xi_{l}\right|\right]^{\alpha_{\tau} /\left[B\left(I_{\mu}, \rho_{\mu}\right)\right]} ; \\
& \left|\hat{\Delta}_{\tilde{b}_{\mu}, k, j, \rho_{\mu}}^{(\tau-1, \kappa)}(\xi, \eta)-\hat{\Delta}_{\tilde{b}_{\mu}, k, j, \rho_{\mu}}^{(\tau-1, \kappa-1)}(\xi, \eta)\right| \leqslant C\left[B\left(I_{\mu}, \rho_{\mu}\right)\right]^{2}\left[\rho_{\mu}^{\kappa j} \sum_{|\beta|=\kappa}\left|\sum_{s=\kappa}^{M} b_{s, \beta} \eta_{s}\right|\right]^{\alpha_{\kappa} /\left[B\left(I_{\mu}, \rho_{\mu}\right)\right]} ;
\end{aligned}
$$

for $\mu=1,2, \ldots ; 1 \leqslant \tau \leqslant N_{0}-1 ; 1 \leqslant \kappa \leqslant M_{0}-1$. 
By (3.3), (3.15)-(3.16), (4.7)-(4.8), Lemma 2.5, Theorems 2.4 and 3.5, and Lemma 2.9, we get

$$
\left\|T_{\tilde{b}_{\mu}} f\right\|_{p}=\left\|\sum_{k, j \in \mathbb{Z}_{-}} \Delta_{\tilde{b}_{\mu}, k, j, \rho_{\mu}}^{\left(N_{0}, M_{0}\right)} * f\right\|_{p} \leq C_{p}\left[B\left(I_{\mu}, \rho_{\mu}\right)\right]^{2}\|f\|_{p},
$$

for every $f \in L^{p}\left(\mathbb{R}^{N} \times \mathbb{R}^{M}\right), \mu=1,2, \ldots$, and for all $p, 1<p<\infty$. Hence, (1.9) follows by (2.3), (4.4), and (4.8).

Finally, a proof of Theorem 1.4 can be obtained using the above estimates and the techniques in [4]. We omit the details.

ACKNOWLEDGMENTS. The publication of this paper was supported by Yarmouk University Research Council. The authors of this paper would like to thank the referees for their valuable remarks.

\section{REFERENCES}

[1] H. Al-Qassem, A. Al-Salman, and Y. Pan, Rough singular integrals with kernels supported by submanifolds of finite type, to appear in Asian J. Math.

[2] __ Singular integrals associated to homogeneous mappings with rough kernels, Hokkaido Math. J. 33 (2004), 1-18.

[3] $\mathrm{H}$. Al-Qassem and Y. Pan, $L^{p}$ estimates for singular integrals with kernels belonging to certain block spaces, Rev. Mat. Iberoamericana 18 (2002), no. 3, 701-730.

[4],$L^{p}$ boundedness for singular integrals with rough kernels on product domains, Hokkaido Math. J. 31 (2002), no. 3, 555-613.

[5] A. Al-Salman, On Rough singular integrals on product domains, preprint, 2004.

[6] A. Al-Salman and Y. Pan, Singular integrals with rough kernels in $L \log L\left(\mathbf{S}^{n-1}\right)$, J. London Math. Soc. (2) 66 (2002), no. 1, 153-174.

[7] J. Duoandikoetxea, Multiple singular integrals and maximal functions along hypersurfaces, Ann. Inst. Fourier (Grenoble) 36 (1986), no. 4, 185-206.

[8] D. Fan, K. Guo, and Y. Pan, Singular integrals along submanifolds of finite type, Michigan Math. J. 45 (1998), no. 1, 135-142.

[9] _ Singular integrals with rough kernels on product spaces, Hokkaido Math. J. 28 (1999), no. 3, 435-460.

[10] D. Fan and Y. Pan, Singular integral operators with rough kernels supported by subvarieties, Amer. J. Math. 119 (1997), no. 4, 799-839.

[11] R. Fefferman, Singular integrals on product domains, Bull. Amer. Math. Soc. (N.S.) 4 (1981), no. 2, 195-201.

[12] R. Fefferman and E. M. Stein, Singular integrals on product spaces, Adv. in Math. 45 (1982), no. 2, 117-143.

[13] Y. S. Jiang and S. Z. Lu, A class of singular integral operators with rough kernel on product domains, Hokkaido Math. J. 24 (1995), no. 1, 1-7.

[14] S. Lu, M. Taibleson, and G. Weiss, Spaces Generated by Blocks, Beijing Normal University Press, Beijing, 1989.

[15] E. M. Stein, Problems in harmonic analysis related to curvature and oscillatory integrals, Proceedings of the International Congress of Mathematicians, Vol. 1, 2 (Berkeley, Calif., 1986), American Mathematical Society, Rhode Island, 1987, pp. 196-221.

Ahmad Al-Salman: Department of Mathematics, Yarmouk University, Irbid, Jordan E-mail address: a1sa7man@yu. edu.jo

Hussain Al-Qassem: Department of Mathematics, Yarmouk University, Irbid, Jordan

E-mail address: husseink@yu.edu.jo 


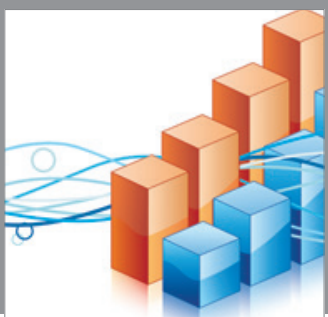

Advances in

Operations Research

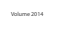

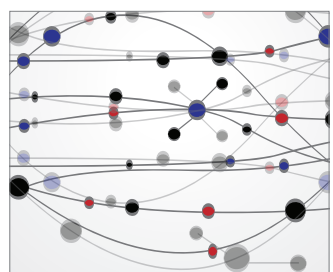

\section{The Scientific} World Journal
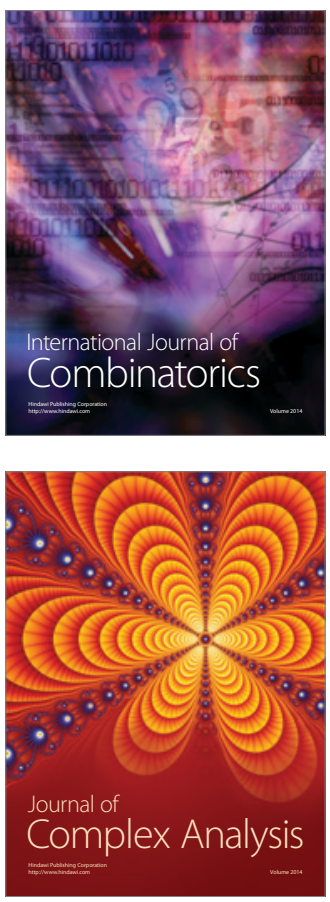

International Journal of

Mathematics and

Mathematical

Sciences
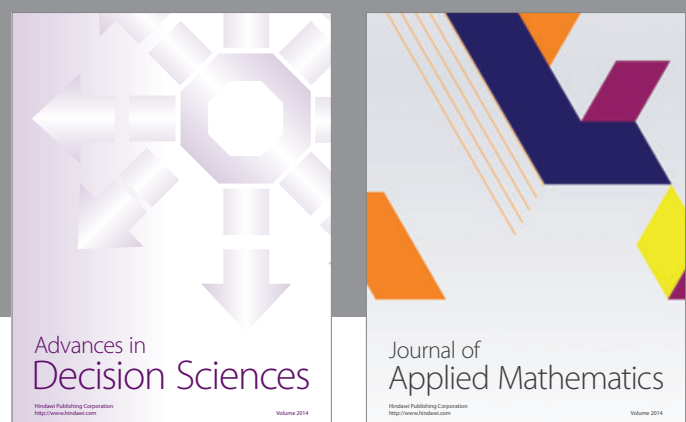

Journal of

Applied Mathematics
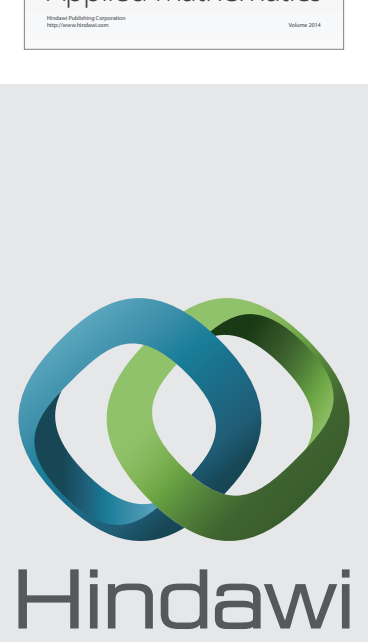

Submit your manuscripts at http://www.hindawi.com
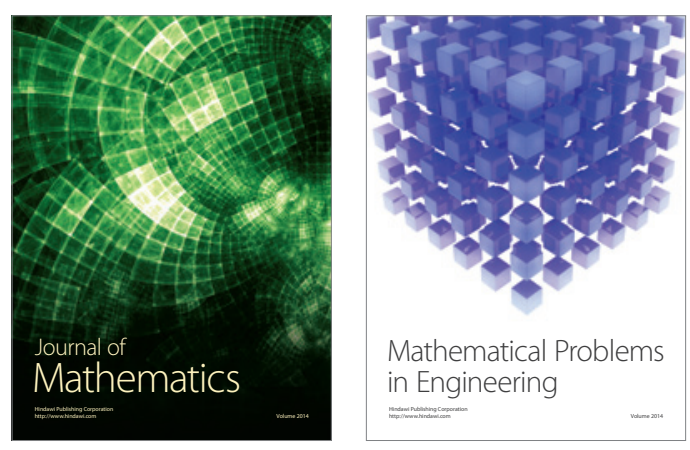

Mathematical Problems in Engineering
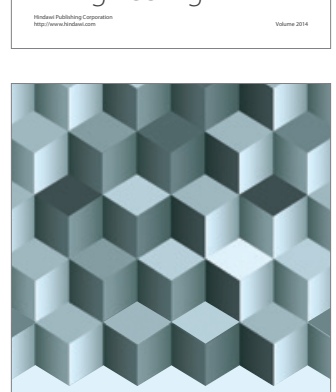

Journal of

Function Spaces
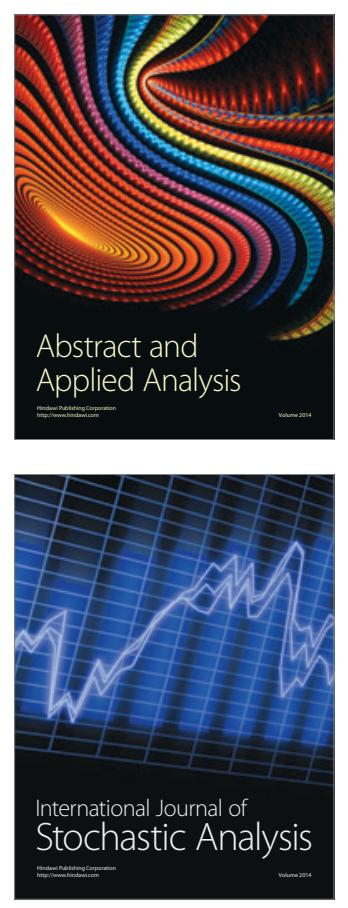

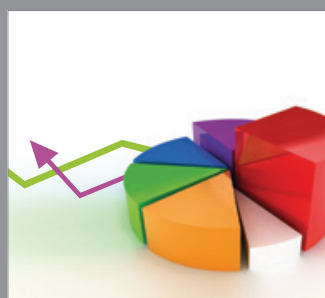

ournal of

Probability and Statistics

Promensencen
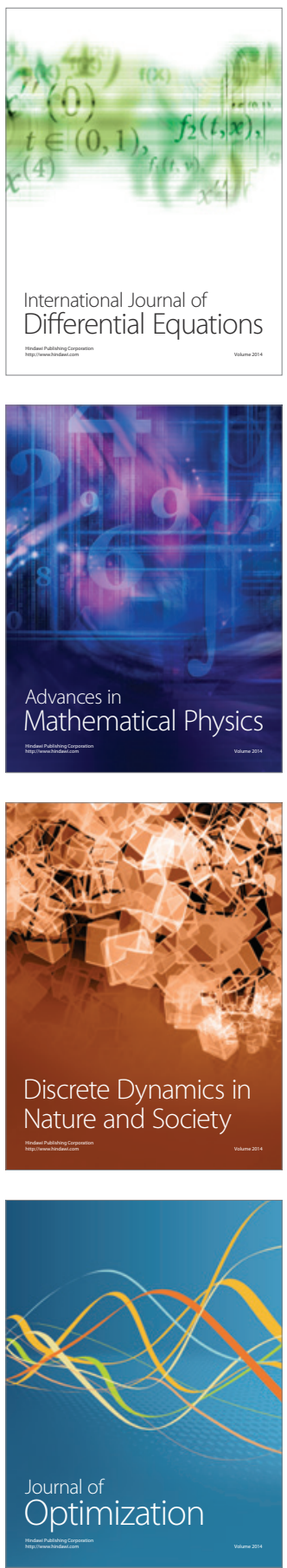